\title{
Correlation of NAFLD fibrosis score and BARD score with ultrasonographic evidence of nonalcoholic fatty liver disease in overweight patients: A prospective study
}

Background: Nonalcoholic fatty liver disease (NAFLD) fibrosis score and BARD score are two of the many noninvasive scoring systems used in the evaluation of the fibrosis in patients with NAFLD biochemically. Ultrasound (USG) is the most common imaging modality for detection of hepatic fibrosis, as it is inexpensive and easily available. Aims: This study attempts to correlate the biochemical and ultrasonographic evidence of fibrosis. It tries to correlate two noninvasive tools for assessing fibrosis in overweight population with NAFLD. Materials and Methods: Prospective study was conducted in which 106 patients participated with BMI; more than 25 underwent ultrasonography for evidence of fatty liver, which was then categorized in three grades, and also scored using biochemical parameters to obtain the NAFLD fibrosis score and BARD score. The scores were then compared with the grades of fatty liver on USG to see for correlation between the two. Results: No statistically significant correlation was found between biochemical evidence of fibrosis and USG evidence of fibrosis in overweight patients of NAFLD. Conclusion: Thus, the biochemical evidence of fibrosis or NAFLD in the form of NAFLD fibrosis score did not correlate with USG evidence of fatty liver. The USG findings of fatty liver may not directly correlate with actual fibrosis in these patients. Context: NAFLD is also an emerging disease in developing countries, which remains silent for years. Noninvasive methods are required for early diagnosis. This study attempts to correlate two noninvasive tools for assessing fibrosis in overweight population of NAFLD. Most of the patients of NAFLD are asymptomatic thus invasive methods are not routinely recommended in them. Thus, it becomes pertinent to study the noninvasive tools extensively for their possible additive use.

Key words: BARD score, imaging fatty liver, nonalcoholic fatty liver disease fibrosis score, nonalcoholic steatohepatitis

Address for the Correspondence:

Dr. Zubin Sharma,

Department of Medicine,

Pad Dr. D. Y. Patil Medical College and Research Hospital, Sant Tukaram Nagar,

Pimpri, Pune - 18, India.

E-mail: drzubinsharma@gmail.com

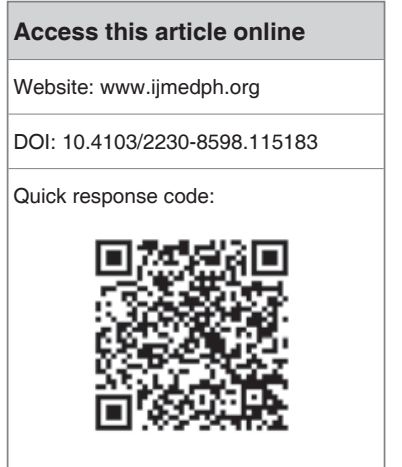

\section{INTRODUCTION}

Nonalcoholic fatty liver disease (NALFD) and nonalcoholic steatohepatitis (NASH) are common often "silent" liver diseases. They resemble alcoholic liver disease, but occur in people who drink little or no alcohol. The major feature in NASH is fatty infiltration of the liver, along with inflammation. Most people with NASH feel well and are not aware that they have a problem. It is commonly an accidental diagnosis when patient is evaluated for some unrelated illness. Asymptomatic transaminase raise is the most common finding in patients of NAFLD.

Nevertheless, NASH can be severe and can lead to cirrhosis, in which the liver is permanently damaged and scarred and can progress to hepatocellular carcinoma.

Currently no non-invasive modality is validated enough to be used for staging of patients with NAFLD and estimating the fibrosis especially in developing countries where advanced imaging studies such as elastography and MRI may not be routinely available.

Ultrasonography (USG) is the most common imaging modality for detection of hepatic fibrosis, being relatively inexpensive and easily available. On USG, fatty liver is seen as a bright liver with echogenicity 
of liver more than the right kidney. Overall, USG has a sensitivity of $60-94 \%$ and a specificity of $84-95 \%$ for detecting fat, ${ }^{[1]}$ NAFLD fibrosis score and BARD score are two of the many noninvasive scoring systems used in the evaluation of the fibrosis in patients of NALFD biochemically.

Angulo et al., ${ }^{[2]}$ developed and validated a simple noninvasive scoring system consisting of routinely measured and easily available clinical and laboratory variables to discriminate between the presence or absence of advanced fibrosis in patients with NAFLD. In a multicenter trial consisting of 480 patients in the derivation cohort and 253 patients in the validation cohort, a low cutoff $(\leq 1.455)$ signified the absence of advanced fibrosis and a high cutoff $(>0.676)$ indicated the presence of advanced fibrosis. ${ }^{[2]}$

The NAFLD fibrosis score consists of six variables, namely age, BMI, AST/ALT ratio, hyperglycemia, platelet count, and serum albumin.

The BARD score was calculated using three easily available variables. These include $\mathrm{BMI}>28 \mathrm{~kg} / \mathrm{m}$ (1 point), AST/ALT $\geq 0.8$ (2 points), and diabetes (1 point). Harrison et al., ${ }^{[3]}$ using this score, showed that a score of 2 to 4 was associated with an odds ratio of 17 (95\% CI, 9.2-31.9) for predicting advanced fibrosis.

NAFLD is an emerging disease, even in developing countries, which remains silent for years. Most of the patients suffering from NAFLD are asymptomatic thus invasive methods are not routinely recommended in them. Thus, it becomes pertinent to study the noninvasive tools extensively for their possible additive use.

Till date no study has directly compared the NAFLD fibrosis score with imaging changes of the fatty liver on USG. This study attempts to correlate the biochemical and ultrasonographic evidence of fibrosis. It tries to correlate two noninvasive tools for assessing fibrosis in overweight population of NAFLD.

\section{MATERIALS AND METHODS}

After clearance from institutional ethics committee and consent, all patients aged 18 and above with body mass index (BMI) of more than 25 and evidence of at least grade 1 fatty liver on ultrasonography (USG) were included in this study.

Patients with common (HBV, HCV) and less common (autoimmune, Wilson's disease, alpha-1-antitrypsin deficiency) liver diseases, hepatic malignancies, infections of biliary tract, alcohol intake of more than $40 \mathrm{~g} /$ week in men and $20 \mathrm{~g} /$ week in women were excluded. Also, patients with a history of systemic illnesses known to cause fatty liver disease, and those who are receiving or have recently received drugs (including herbal medicines) known to raise ALT, AST to cause fatty liver disease were excluded.

Total 106 patients fulfilling the inclusion-exclusion criteria were included in the study. It was a prospective study done at a tertiary care hospital between July 2010 and September 2012 and data were generated as per the performa and patient's records.

\section{Definitions}

\section{Nonalcoholic fatty liver disease}

Nonalcoholic fatty liver disease (NAFLD) was defined as (a) there is evidence of hepatic steatosis, either by imaging or by histology and (b) there are no causes for secondary hepatic fat accumulation such as significant alcohol consumption, use of steatogenic medication or hereditary disorders

\section{Overweight}

Patients with body mass index (BMI) of more than 25 calculated as mass (in kilogram) per height (in meters). It was taken as per the World Health Organization (WHO) international classification.

\section{Fatty liver}

Fatty liver was defined by the presence of at least two of three abnormal findings on abdominal ultrasonography. The USG of all patients was done on the same USG machine and in similar lighting conditions.

Diffusely increased echogenicity (bright) liver with liver echogenicity greater than kidney or spleen, hepatic vascular blurring, and deep attenuation of ultrasound signal in posterior liver and diaphragm.

Fatty liver was graded by following means:

Grade 1 (Mild) - minimal diffuse increase in hepatic echogenicity with normal visualization of diaphragm and intrahepatic vessel borders

Grade 2 (Moderate) - moderate diffuse increase in hepatic echogenicity with slightly impaired visualization of intrahepatic vessels and diaphragm

Grade 3 (Severe) - marked increase in echogenicity with poor penetration of posterior segment of right lobe of liver and poor or no visualization of hepatic vessels and diaphragm.

Metabolic syndrome - metabolic syndrome was defined as per the International Diabetes Federation Guidelines (IDF). ${ }^{[4]}$

On inclusion in the study, the patient were assessed by anthropometric, clinical, and biochemical parameters. The data generated were then used to score the patients using the NAFLD fibrosis score and the BARD score. Data were also analyzed to categorize patients with metabolic syndrome using the above said IDF guidelines.

The various variables assessed were as follows:

1. Anthropometry: Height, weight, BMI, waist circumference, hip circumference, waist to hip ratio

2. Blood pressure measurement

3. Biochemical: S. Bilirubin, Serum ALT, AST and ALP, S. Proteins including albumin, globulin, S. GGT, S. Ferritin, and fasting serum lipids

4. Complete hemogram.

\section{NAFLD fibrosis score ${ }^{[2]}$}

The NAFLD fibrosis score was calculated according to the following formula:

$-1.675+0.037 \times$ age $($ years $)+0.094 \times$ BMI $\left(\mathrm{kg} / \mathrm{m}^{2}\right)+$ $1.13 \times \mathrm{IFG} /$ diabetes $($ yes $=1$, no $=0)+0.99 \times \mathrm{AST} / \mathrm{A} \mathrm{LT}$ ratio $-0.013 \times$ platelet $(\times 109 / \mathrm{L})-0.66 \times$ albumin $(\mathrm{g} / \mathrm{dL})$. 
NAFLD score $\leq 1.455=$ less probability of fibrosis

NAFLD score $-1.455-0.675=$ indeterminate score

NAFLD score $>0.675=$ high probability of fibrosis.

\section{$B A R D$ score ${ }^{[3]}$}

BARD score was calculated as weighted sum: BMI $\geq 28=1$ point + AAR of $\geq 0.8=2$ points $+\mathrm{DM}=1$ point. $\mathrm{BARD}$ score of more the 2 indicates fibrosis.

SPSS 16.0 was used for statistical analysis. Data were analyzed using statistical analysis tools like ANOVA for multivariate analysis. F and $P$ values were obtained for estimating relation between the scores and increasing grade of fatty liver on imaging.

\section{RESULTS}

Majority of the study participants $(n=35)$ were in the age group of 41-50. There were 14 participants between 18 and 30 years of age. There were seven between the age group of 71-80. Thus, the study group was well represented from all age groups. Sixty nine out of 106 study participants were females and 37 were males. There were twenty seven patients with grade 1 fatty liver in BMI between 25-29.9, 32 in BMI range of 30-34.5, five in BMI range in 35-39.9 and none in more than 40 . There were ten patients with fatty liver grade 2 in the BMI range of 25-29.9, 15 in the BMI range of 30-34.9, six in the BMI range of 35-39.9 and three in the BMI range more than 40 . There were total five cases of grade 3 fatty liver, one each in the BMI range of 30-34.9 and 35-39.9 and three in BMI range of more than 40 . Thus, in total, $63 \%$ study participants had grade 1 fatty liver on USG and $32 \%$ had grade 2 fatty liver.

The mean NAFLD fibrosis score with grade 1 fatty liver was -0.44 , grade 2 fatty liver had mean score of -0.13 , and grade 3 fatty liver had mean score of 0.15 . The $F$ value was 0.99 for the relation between Imaging grades and NAFLD fibrosis score. The $P$ value was 0.12 [Figure 1].

The mean BARD score with grade 1 fatty liver was $2.81 \pm 0.74$, grade 2 fatty liver had mean score of $2.94 \pm 0.95$, and grade 3 fatty liver had mean score of $2.60 \pm 0.89$. The $F$ value obtained was 0.53 , thus, rendering the relation not significant statistically. The $P$ value was 0.18 [Figure 2].

\section{DISCUSSION}

This study was conducted to correlate biochemical and imaging in patients with NAFLD who were overweight with BMI of more than 25. One hundred and six patients were included in this study and they were assessed in terms of imaging evidence of fatty liver, biochemical parameters, and NAFLD fibrosis score and BARD score. The imaging changes were subsequently compared with the aforementioned noninvasive scores of NAFLD to correlate whether imaging changes match with biochemical changes.

No study has till date directly compared the NAFLD fibrosis score with imaging changes of the fatty liver on USG.

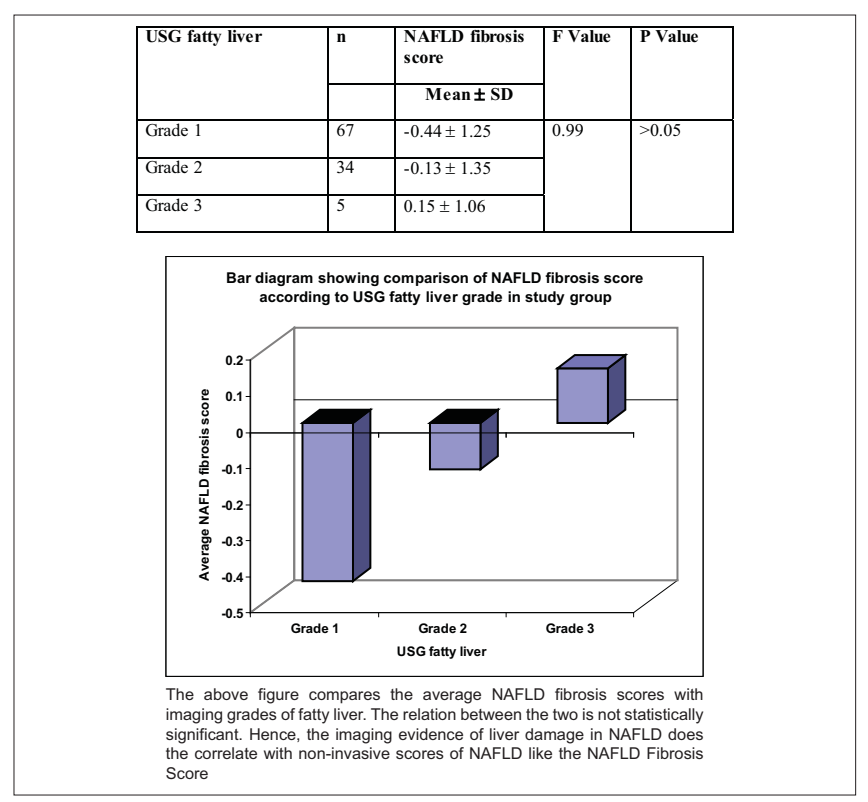

Figure 1: Comparison of NAFLD Fibrosis score according to USG fatty liver grade in study group

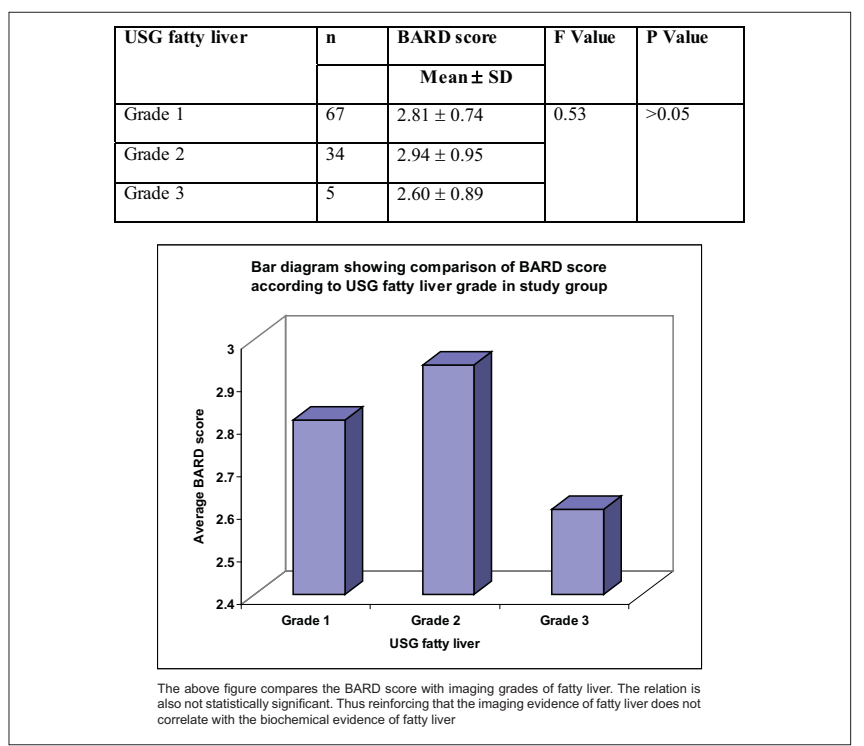

Figure 2: Comparison of BARD score according to USG fatty liver grade in study group

In this study, the mean NAFLD fibrosis score with grade 1 fatty liver was -0.44 , grade 2 fatty liver had mean score of -0.13 , and grade 3 fatty liver had mean score of 0.15 . The $F$ value was 0.99 for the relation between imaging grades and NAFLD fibrosis score.

This relation was not found to be statistically significant. Thus, the biochemical evidence of fibrosis or NAFLD in the form of the NAFLD fibrosis score did not correlate with imaging evidence of fatty liver. Thus, imaging findings of fatty liver may not directly correlate with actual fibrosis in these patients.

It was also attempted to correlate the BARD score with the imaging 
studies. The $F$ value obtained was 0.53 , thus, rendering the relation not significant statistically. Thus, the BARD score did not correlate with imaging evidence of fibrosis like the NAFLD fibrosis score.

Out of two noninvasive scores, though both statistically non significant, NAFLD fibrosis score correlated better with the imaging changes as compared to the BARD score.

This result is to be taken into account in respect to other studies that have validated the ultrasonography as a modality of screening of fatty liver. Overall, USG has a reported sensitivity of $60-94 \%$ and a specificity of $84-95 \%$ for detecting fat, ${ }^{[1]}$ but combined fat and fibrosis can show up a hyperechoic liver in $98.7 \%$ of patients known as 'fatty fibrotic pattern'. Sensitivity depends on the amount of fat in liver, however, both sensitivity and specificity are poor in morbid obesity. ${ }^{[5]}$

Patients with severe steatosis have marked increase in echogenicity and poor posterior penetration and poor or nonvisualization of the diaphragm and the intrahepatic vessels. ${ }^{[5]}$ However, ultrasound has the disadvantage of being subjective and less sensitive and specific in patients with obesity. Hence, in patients, like in this study, who have higher BMI levels, USG may not correctly reflect the amount of fibrosis.

Also, USG being operator dependent may have contributed to this result.

Another point raised with this study is the possible use of biochemical and imaging study together as additive modality for monitoring and estimation of fibrosis in NAFLD patients. Though this study was not designed to look into this aspect, but additive role of these two noninvasive modalities should be looked into by carefully designed studies for possible use.

\section{CONCLUSION}

No statistically significant correlation was found between biochemical evidence of fibrosis and USG evidence of fibrosis in overweight patients of NAFLD.

Thus, the biochemical evidence of fibrosis or NAFLD in the form of NAFLD fibrosis score did not correlate with imaging evidence of fatty liver. The USG findings of fatty liver may not directly correlate with actual fibrosis in these patients. The study raises question of estimation of fibrosis in patients of NAFLD using USG and biochemical parameters alone and possible use of both modalities together as additive evidence. Future studies planned to look into the noninvasive diagnosis, staging and monitoring of patients of NAFLD using various modalities together could yield better results.

\section{REFERENCES}

1. Mehta SR, Thomas EL, Bell JD, Johnston DG, Taylor-Robinson SD. Non-invasive means of measuring hepatic fat content. World J Gastroenterol 2008;14:3476-83.

2. Angulo P, Hui JM, Marchesini G, Bugianesi E, George J, Farrell GC, et al. The NAFLD fibrosis score: A noninvasive system that identifies liver fibrosis in patients with NAFLD. Hepatology 2007;45:846-54.

3. Harrison SA, Oliver D, Arnold HL, Gogia S, Neuschwander Tetri BA. Development and validation of a simple NAFLD clinical scoring system for identifying patients without advanced disease. Gut 2008;57:1441-7.

4. Alberti KG, Zimmet P, Shaw J. Metabolic syndrome: A new world-wide definition. A Consensus Statement from the International Diabetes Federation. Diabet Med 2006;23:469-80.

5. Saverymuttu SH, Joseph AE, Maxwell JD. Ultrasound scanning in the detection of hepatic fibrosis and steatosis. Br Med J (Clin Res Ed) 1986;292:13-5.

How to cite this article: Kakrani AL, Sharma ZD, Thind SS, Gokhale VS. Correlation of NAFLD fibrosis score and BARD score with ultrasonographic evidence of nonalcoholic fatty liver disease in overweight patients: A prospective study. Int J Med Public Health 2013;3:111-4.

Source of Support: The study was possible by generous research grants from Dr. D. Y. Patil Vidyapeeth (Deemed University), Conflict of Interest: None declared. 